\title{
Turbulence et séparation
}

\section{Turbulence and separation}

\author{
Y. Lecoffre \\ Ingénieur conseil
}

La turbulence intervient comme facteur limitant les performances des séparateurs gravitaires ou tournants. Pratiquement, le taux de turbulence admissible doit être tel que les fluctuations moyennes de vitesse soient de l'ordre de 5 fois inférieures aux vitesses de chute.

On présente deux types de séparateurs, l'un gravitaire, l'autre cyclonique dans lesquels la suppression quasi complète de la turbulence a permis d'améliorer les diamètres de coupure de façon spectaculaire. Dans le cas du séparateur gravitaire, on a créé un écoulement laminaire. Dans le cas du cyclone tournant, on a rendu négligeable l'action néfaste de la turbulence en faisant tourner les parois extérieures.

Turbulence acts as a factor limiting the performances of whirling or gravitative separators. In practice, the admissible rate of turbulence must be such that the average velocity variations are approximatly five times less than the fall velocities.

There are two types of separators, namely, one being gravitative, the other cyclonic, in which the almost complete suppression of turbulence has enabled the cut-off diameters to be spectacularly improved. As regards the gravitative separator, a laminar flow has been created. As regards the whirling cyclone, the disastrous effect of turbulence is significantly reduced by causing the exterior walls to rotate.

\section{Introduction}

Le C.E.R.G. (Centre d'études et de recherches de Grenoble) a été confronté à plusieurs problèmes de séparation de phases, soit dans le cadre de réalisations d'ensembles complexes, soit dans le but de réaliser des appareils industriels.

Dans tous les cas, on a cherché à minimiser la dimension de l'appareil, en jouant, pour ce faire sur les éléments suivants :

- L'entrée du séparateur. Il est indispensable d'assurer une équirépartition des débits pour éviter des recirculations totales ou partielles.

- Les éléments séparateurs proprement dits. Il y règne une compétition entre la turbulence, qui tend à homogénéiser les concentrations et le champ d'accélération, quel que soit son type, qui est l'élément moteur de la séparation.

- Les sorties. Il est souvent difficile d'obtenir une pureté parfaite des phases séparées s'il n'y a pas de grossissement des globules par coalescence.

Après un bref rappel de quelques grandeurs caractérisant le fonctionnement de divers séparateurs à gravité naturelle ou forcée, on donne deux exemples de réalisation :

- Le décanteur à bulles du G.T.H. (Grand tunnel hydrodynamique) du Vaudreuil permet de traiter jusqu'à 32 $\mathrm{m} / \mathrm{s}$ de débit. Son diamètre de coupure est, dans ces conditions, de l'ordre de $100 \mu$. Il sépare l'air contenu dans des émulsions grossières jusqu'à des taux de vide de $10 \%$. - Les cyclones séparateurs d'huile dispersée dans l'eau. Dans une première réalisation, les diamètres de coupure des microgouttes d'huile dans l'eau étaient de l'ordre de $100 \mu$. On montre qu'en supprimant presque complètement la turbulence dans l'appareil, on obtient, toutes choses égales par ailleurs, des performances de l'ordre de 20 à 30 fois supérieures, tout en améliorant notablement la souplesse du système.

LA HOUILLE BLANCHE/N 7/8-1987 


\section{Vitesses terminales et turbulence}

\subsection{Vitesses terminales}

Dans un fluide $(f)$ au repos dans un champ d'accélération $\vec{\gamma}$ un globule $(g)$ de densité différente tend vers une vitesse limite qui, pour les faibles nombres de Reynolds $(R<2)$ s'écrit :

$$
\vec{W}=\frac{1}{18} \cdot \vec{\gamma} \cdot \frac{\Delta \rho \cdot d^{2}}{\mu}
$$

avec $\Delta \rho=\left(\rho_{s}-\rho_{\ell}\right)$

$\mu$ : la viscosité cinématique du fluide $f$ $d$ : le diamètre du globule.

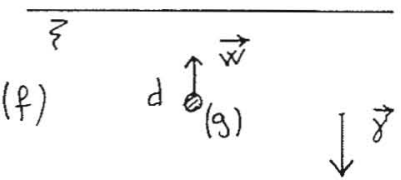

1. Séparation dans un champ d'accélération.

Il s'agit là de la formule la plus couramment admise, que l'on utilise pratiquement pour dimensionner un séparateur (Réf. 1).

La vitesse terminale théorique de globules contenus dans un fluide donné ne peut être modifiée que par une modification de l'accélération.

On donne, à titre d'exemple, les vitesses terminales d'une goutte d'huile $\left(\Delta \rho=50 \mathrm{Kg} / \mathrm{m}^{3}\right)$ de $20 \mu$ de diamètre dans de l'eau froide $\left(\mu=10^{-3}\right)$ dans différentes situations.

En décantatation naturelle $W=0,01 \mathrm{~mm} / \mathrm{s}$.

Dans un écoulement tournant, l'accélération centrifuge locale est telle que :

$$
|\vec{\gamma}|=\frac{V_{i}^{2}}{r}
$$

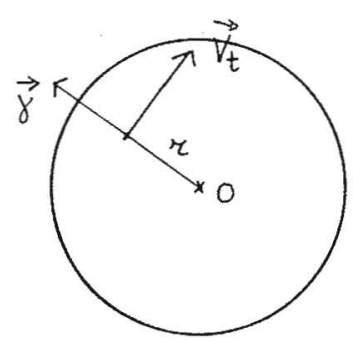

2. Décantation en écoulement tournamt.

Tout en restant dans des ordres de grandeur de vitesse et de dimensions raisonnables, on obtient aisément des accélérations très supérieures à celle de la pesanteur.
Par exemple, en prenant la même goutte pour référence :

$$
\begin{aligned}
& \text { pour } V t=5 \mathrm{~m} / \mathrm{s} \\
& r=2,510^{-2} \mathrm{~m} \\
& |\vec{\gamma}|=1000 \mathrm{~m} / \mathrm{s}^{2} \quad W=1 \mathrm{~mm} / \mathrm{s} \\
& \text { pour } V t=10 \mathrm{~m} / \mathrm{s} \\
& \text { et } r=2,510^{-2} \mathrm{~m} \\
& |\vec{\gamma}|=4000 \mathrm{~m} / \mathrm{s}^{2} \quad W=4 \mathrm{~mm} / \mathrm{s}
\end{aligned}
$$

On peut, très grossièrement, estimer que le volume d'un décanteur est proportionnel à la vitesse de chute $W$. Les ordres de grandeur ci-dessus montrent l'intérêt évident d'utiliser des écoulements tournants pour réduire, sinon la complexité, du moins l'encombrement des appareils.

\subsection{Les effets de la turbulence}

La turbulence a tendance à réhomogénéiser les concentrations. En l'absence de forces extérieures, si, dans un écoulement turbulent, existe un gradient de concentration $\partial C / \partial y$ et si la diffusion turbulente est localement égale à $D t$, le débit massique traversant une surface $d S$ est tel que :

$$
\frac{d m}{d t} \sim D t \cdot \frac{\partial C}{\partial y} \cdot d S
$$

Ce transport se fait des zones de forte concentration vers les zones de faible concentration.

Dans un décanteur règne souvent une certaine turbulence, qui a pour effet de remettre en suspension les globules à séparer.

Dans le cas, par exemple, d'un décanteur gravitaire, la séparation des espèces ne peut se faire que si la vitesse de chute est nettement supérieure aux fluctuations turbulentes. On considère souvent que, si le taux de turbulence est égal à $\tau$, le séparateur fonctionne dans de bonnes conditions si $W>k \tau U$ avec $k$ de l'ordre de 5, $U$ étant la vitesse moyenne locale d'entraînement du fluide porteur.

De manière équivalente dans un séparateur, les vitesses minimales de décantation doivent être de l'ordre de 5 fois les fluctuations moyennes de vitesse dans le sens de la décantation.

\subsection{Conclusion pratique}

Si l'on revient à l'exemple de la goutte de $20 \mu$ donné ci-dessus, on en conclut que la séparation est quasi impossible à réaliser en gravitaire, à moins d'opérer en écoulement laminaire.

Dans le cas d'un écoulement tournant, type cyclone, qui est dans pratiquement tous les cas classiques turbulent, séparer une goutte de $20 \mu$ obligerait à utiliser des vitesses tangentielles extrèmement élevées. Là encore, il est nécessaire de relaminariser l'écoulement, ce qui impose l'emploi d'internes complexes, et n'est actuellement réalisable que dans des centrifugeuses, donc en écoulement tournant de type rotation solide, moins efficace qu'un cyclone.

Le choix d'un appareil de séparation résulte toujours de compromis où interviennent la compacité, le diamètre de coupure, la perte de charge et le coût. Les critères à retenir doivent également tenir compte, et ceci d'autant plus que les appareils sont performants, des fluctuations de débit, de concentration et de distributions granulomé- 


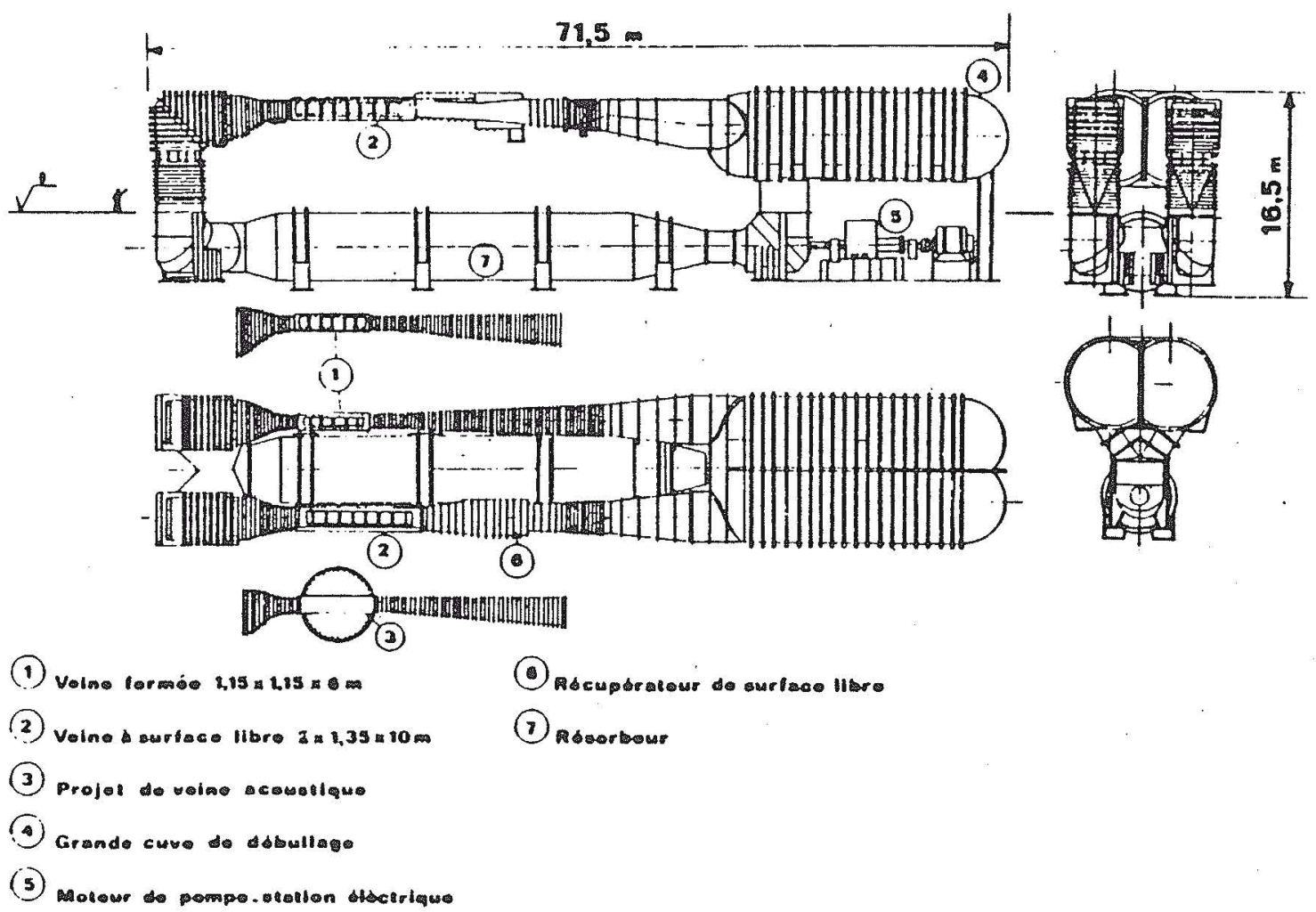

3. Schéma du Grand ummel hydrod!namique. Bassin des carènes. Projet Nelrtec.

triques. La part de la régulation aura donc de plus en plus tendance à augmenter dans les installations de séparation.

Il n'en reste pas moins que l'optimisation d'un séparateur dans ses conditions les plus critiques de fonctionnement, passe par l'adéquation du taux de turbulence résiduel au champ d'accélération disponible.

\section{La cuve aval du G.T.H.}

\subsection{Le problème posé}

Un tunnel de cavitation de très grandes dimensions a été construit par Alsthom pour la Marine Nationale au Vaudreuil près de Rouen; le débit maximal dans la veine d'essais à surface libre est de $32,4 \mathrm{~m}^{3} / \mathrm{s}$ (Fig. 3).

Dans ce tunnel on assure un contrôle des germes de cavitation. Ceci suppose entre autres que l'on soit en mesure d'éliminer dans la cuve aval toute bulle de gaz de diamètre supérieur à $100 \mu$. Ce tunnel est par ailleurs susceptible de véhiculer de très grandes quantités d'air, de l'ordre de $10 \%$ du débit volume principal. L'air contenu dans cette émulsion doit également être éliminé dans la cuve aval.

Il a été nécessaire de concevoir et réaliser un décanteur susceptible de travailler avec des émulsions très différentes, pénétrant éventuellement simultanément dans l'appareil.

Typiquement, l'émulsion de grosses bulles a une granulométrie moyenne de 3 à $4 \mathrm{~mm}$ et un taux de vide de quelques pour cent. Celle de fines bulles, pour autant qu'on puisse encore parler d'émulsion, contiendra une centaine de bulles par $\mathrm{cm}^{3}$, de quelques dizaines à quelques centaines de microns de diamètre.

On a, bien entendu, cherché à réduire la dimension du décanteur, compte tenu du fait que sa hauteur minimale était de l'ordre de $4 \mathrm{~m}$, et que la pression maximale dans l'appareil pouvait atteindre 5 bars absolus.

\subsection{La solution retenue}

\section{a) Les éléments décanteurs}

La vitesse ascentionnelle des bulles contenues dans l'émulsion grossière est de l'ordre de $30 \mathrm{~cm} / \mathrm{s}$. Il est donc possible de les séparer dans un décanteur lamellaire 
fonctionnant en écoulement turbulent avec une vitesse axiale maximale de l'ordre de $40 \mathrm{~cm} / \mathrm{s}$. Dans le GTH, on a disposé un premier étage de toits séparés chacun de $70 \mathrm{~mm}$ et munis à leur extrémité de collecteurs, eux-mêmes reliés à une cheminée. La figure 4 donne un schéma de l'ensemble. On notera que la cheminée est fermée à sa partie inférieure et que des diaphragmes ont été disposés à la sortie des collecteurs. Cette solution a permis d'éviter qu'un entrainement d'eau par air-lift se produise dans les cheminées, ce qui aurait perturbé leur fonctionnement.

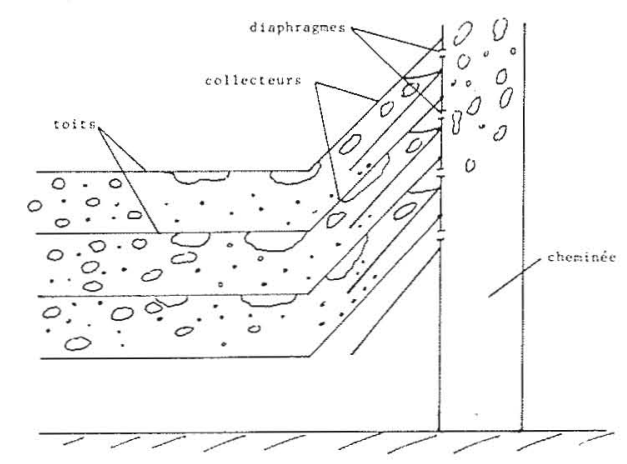

4. Décanteurs primaires G.T.H.

Le premier étage permet d'éliminer de l'écoulement les bulles millimétriques. Pour les petites bulles, de rayon inférieur à $250 \mu$ environ, il n'est pas possible d'utiliser ce procédé. L'écoulement étant turbulent, la décantation ne peut plus se produire, comme l'ont confirmé des essais préliminaires.

On a donc construit un second étage, formé de nids d'abeilles de maille $D$ égale à $8 \mathrm{~mm}$. Pour qu'une décantation des microbulles se produise, il est alors impératif que l'écoulement soit laminaire. Des essais nous ont conduits à admettre un nombre de Reynolds maximal de 2400 . La détermination de la surface frontale du décanteur résulte de l'application de cette règle. On obtient en effet une vitesse maximale d'écoulement telle que pour de l'eau froide $V_{\max }=(2400 / v D)=0,3 \mathrm{~m} / \mathrm{s}$.

Compte tenu d'éventuelles inhomogénéités de vitesse dans l'ensemble du décanteur, la vitesse moyenne maximale a été fixée à $0,2 \mathrm{~m} / \mathrm{s}$. La surface totale de l'appareil est donc $S=32,4 / 0,2=162 \mathrm{~m}^{2}$.

La hauteur fixée par d'autres critères, étant de $4,5 \mathrm{~m}$, la longueur des décanteurs est de $36 \mathrm{~m}$.

Dans le nid d'abeilles, les bulles remontent vers la paroi supérieure. On constate expérimentalement qu'il s'y produit une coalescence. A la sortie du nid d'abeilles, les bulles ainsi formées sont millimétriques. Des essais sont là aussi en cours, destinés à comprendre les mécanismes fins du processus.

Il reste alors à disposer à l'aval du nid d'abeilles, un second décanteur lamellaire, identique à celui du premier étage pour récupérer les bulles coalescées et les évacuer au travers des cheminées (Fig. 5).

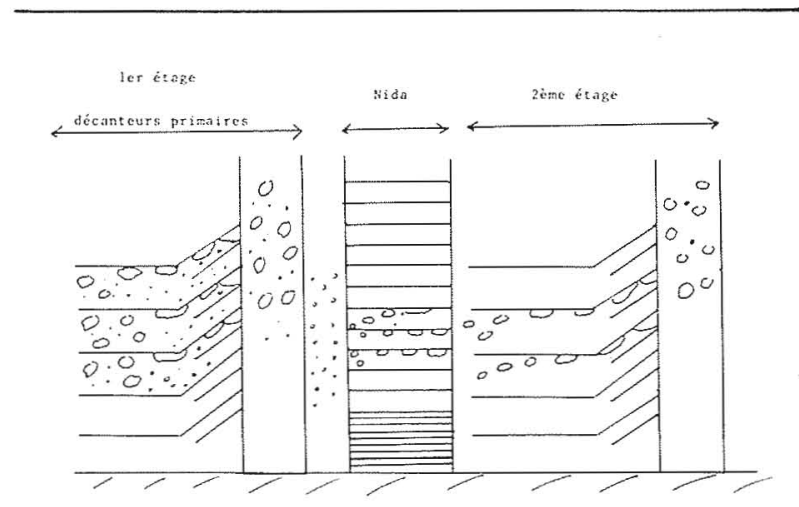

5. Schéma d'ensemble du décameur G.T.H.

\section{b) L'alimentation du décanteur}

Dans le cas du G.T.H., l'eau qui traverse le décanteur provient d'une veine d'essais, dans laquelle la vitesse maximale d'écoulement est, suivant les cas, de $12 \mathrm{~m} / \mathrm{s}$ ou de $20 \mathrm{~m} / \mathrm{s}$. Il est nécessaire de ralentir cet écoulement pour le faire pénétrer uniformément dans l'ensemble de toits, soit sur $160 \mathrm{~m}^{2}$. La divergence de l'écoulement est dans le cas le plus critique, de 120 , entre la veine d'essais et le décanteur. Cette alimentation uniforme doit, bien entendu, être réalisée avec un minimum de pertes de charge.

La figure 6 donne un schéma de la cuve aval définitive. L'eau provenant de l'une ou l'autre des veines d'essais est d'abord ralentie dans un diffuseur simple, puis un diffuseur emboîté. On pénètre dans la cuve aval au travers de deux coudes successifs à la sortie desquels la vitesse maximale est de $2 \mathrm{~m} / \mathrm{s}$. Le débit est alors partagé en deux parties dans la cuve et la vitesse axiale moyenne dans les dièdres d'alimentation est de $1 \mathrm{~m} / \mathrm{s}$. L'entrée dans les décanteurs se fait au travers de grilles spéciales développées par J. Poux, qui ont pour fonction de créer des pertes
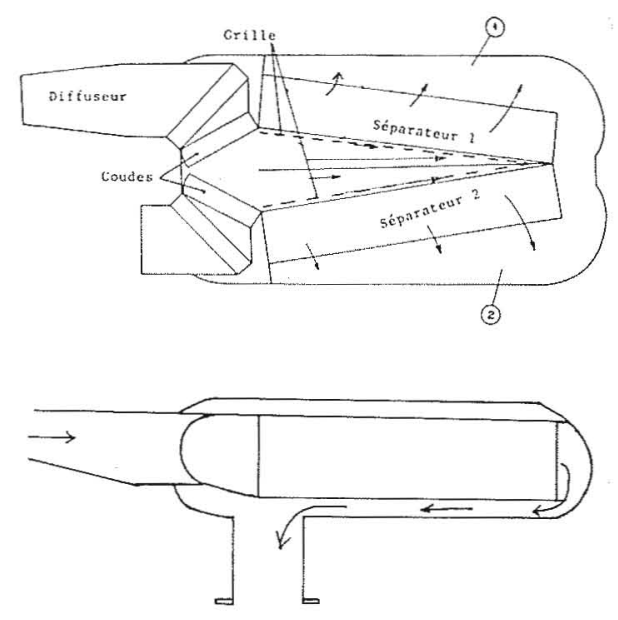

6. Cure aral du G.T.H. - Organisation des écoulements. 
de charge tendant à équirépartir les débits tout en redressant l'écoulement.

La mise au point de l'alimentation des décanteurs a nécessité l'utilisation d'un modèle réduit à échelle $1 / 10$. Plusieurs modifications ont été nécessaires pour éviter, dans un premier temps, la création de courants de retour dans les nids d'abeilles, puis dans un deuxième temps, pour obtenir une homogénéisation des vitesses telle qu'elles ne soient en aucun point supérieures à $30 \mathrm{~cm} / \mathrm{s}$.

La sortie du gaz s'effectue, au travers des cheminées, au-dessus des toits supérieurs de la cuve. Celle de l'eau suit le parcours schématisé par des flèches. Cette configuration d'écoulement a été retenue pour intégrer le retour à la cuve et en diminuer ainsi la longueur et le volume.

\section{Le cyclone tournant}

Le pétrole brut sortant des puits contient, outre des hydrocarbures liquides, du gaz, des solides et de l'eau. La proportion d'eau peut atteindre jusqu'à $90 \%$ du mélange. Cette eau doit être rejetée en milieu naturel selon des normes très sévères, la teneur maximale en hydrocarbures résiduels étant de l'ordre de 10 à 100 ppm suivant les sites. Il est donc impératif de disposer de séparateurs de phases efficaces et, si possible, peu encombrants et légers spécialement dans le cas de traitement sur plateformes en mer.

\subsection{Les cyclones}

Dans leur principe, les cyclones sont le séparateur idéal, spécialement dans le cas d'une phase légère dispersée dans un liquide lourd.

La figure 7 compare les champs de vitesse tangentielle et d'accélération produits par une centrifugeuse (rotation en masse) et par un cyclone (rotation en vortex).
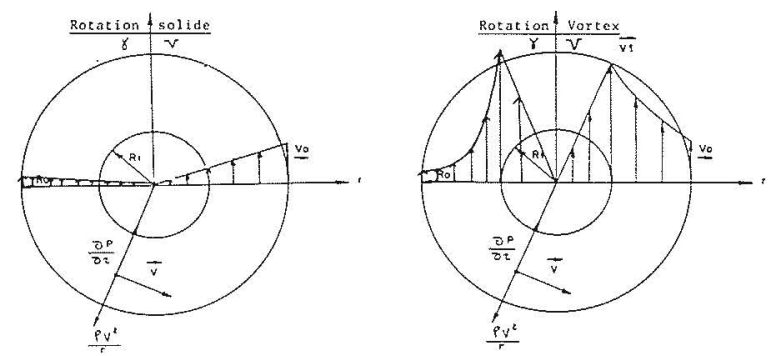

7. Champ de vitesse tangentielle pour une rotation solide et une rotation en romes.

En termes d'efficacité, si l'on compare le temps que met un globule pour atteindre, en partant de la paroi extérieure, le rayon $\mathrm{R} / 3$, on trouve qu'il est 5 fois inférieur dans le cas d'un hydrocyclone à ce qu'il est dans une centrifugeuse.
On peut dès lors s'étonner qu'avec un tel facteur de mérite, ces appareils soient très peu utilisés en séparation liquide-liquide. La raison en est peut-être que, dans les cyclones conventionnels, la turbulence diminue l'efficacité dans des proportions considérables.

\subsection{Cyclone fixe}

Neyrtec a développé, en 1984, un cyclone à paroi fixe, dont le schéma est donné sur la figure 8. L'étude a été réalisée en collaboration avec la C.F.P.

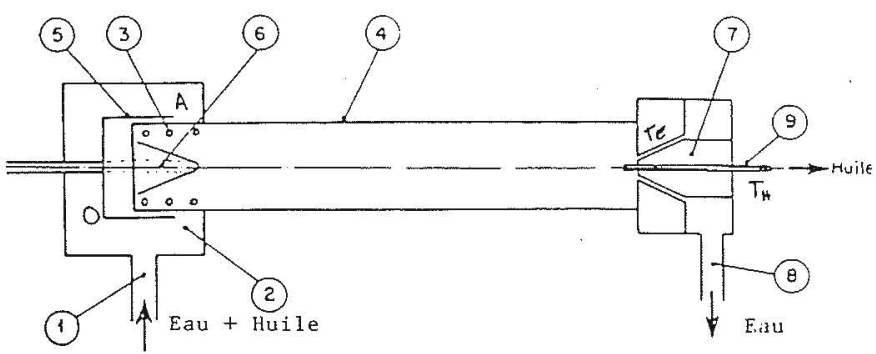

8. Schéma du cuclone five.

L'eau pénètre dans le tube séparateur cylindrique au travers d'orifices tangentiels équirépartis, dont le nombre peut être modifié au moyen d'un obturateur mobile.

Dans le but de stabiliser l'écoulement en entrée, on a disposé une forme conique (6) dans la zone amont du tube séparateur, solution qui s'est avérée très efficace.

La sortie de la phase légère s'effectue par une tuyauterie axiale (9), le prélèvement pouvant être réalisé à l'amont ou à l'aval de l'appareil. Le fluide porteur, l'eau dans ce cas d'application, est évacuée au travers d'une forme destinée à récupérer une partie de l'énergie cinétique, constituée d'un espace entre deux cones d'angles légèrement différents (7).

Cette configuration d'écoulement a permis d'obtenir des performances en séparation intéressantes, comparables à celles obtenues par d'autres expérimentateurs (Réf. 2). Pour un appareil de diamètre interne égale à $60 \mathrm{~mm}$, de longueur $1 \mathrm{~m}$, fonctionnant sous 3 bars, avec un débit de $3 \mathrm{~m}^{3} / \mathrm{h}$ toute goutte de diamètre supérieur à $150 \mu$ était séparée. L'efficacité de séparation dans les classes granulométriques inférieures était très variable. Elle tombait par exemple à $75 \%$ à $50 \mu$ et à $40 \%$ à $25 \mu$ (Fig. 9, page suiv.)

Les différences très importantes entre les performances théoriques et les résultats obtenus s'expliquent par la turbulence induite dans le cyclone, qui perturbe totalement son fonctionnement.

- Les jets tangentiels pénètrent dans le cyclone à une vitesse légèrement inférieure à la vitesse tangentielle locale. La dissipation qui en résulte a pour effet de diminuer la circulation. Elle peut, par ailleurs être source de fractionnement des gouttes.

- Le frottement sur les parois freine la rotation de manière très significative. De ce fait, les accélérations diminuent très rapidement à mesure que l'on avance vers 


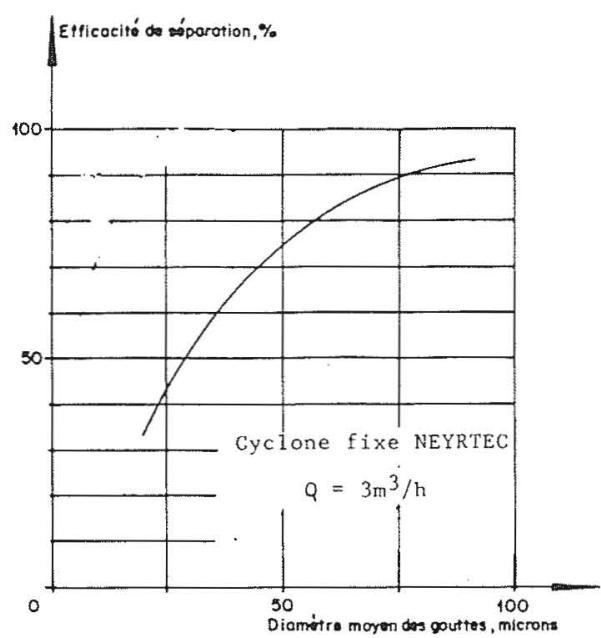

9. Efficacité de séparation du ciclone fixe.

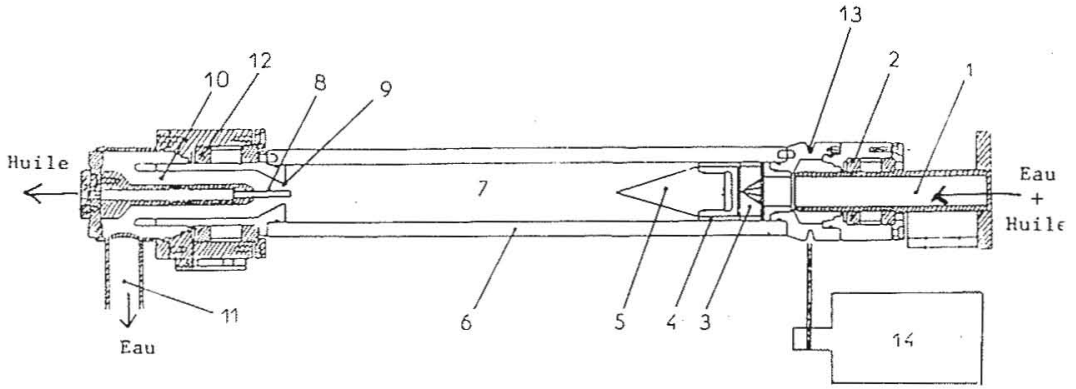

10. Schéma du ciclone tournant. la sortie. Il ne sert plus à rien d'augmenter la longueur du cyclone au-delà d'une certaine valeur critique.

- Les frottements se traduisent, bien entendu, par une augmentation significative du taux de turbulence, ce qui explique que l'efficacité réelle est inférieure à l'efficacité théorique basée sur la distribution mesurée de vitesse moyenne. Cette distribution est d'ailleurs assez différente de celle d'un vortex théorique (loi de vitesse en $(1 / r)^{0 . x}$ au lieu de $(1 / r)$.

- Ces dissipations sont sources d'instabilités parfois spectaculaires du barreau central. En l'absence de cone stabilisateur, le barreau d'huile pouvait se déplacer pratiquement jusqu'à la paroi extérieure de l'appareil.

- Enfin, les frottements sont à l'origine d'écoulements secondaires qui détruisent l'homogénéité des vitesses axiales. Bien que, dans certains cas, ce phénomène puisse théoriquement améliorer la séparation, l'analyse faite à partir d'hypothèses sur la distribution de vitesses axiales montre que ce n'était probablement pas le cas lors de nos essais.

- Si l'on ajoute à ces remarques le fait que la gamme de débit de fonctionnement d'un cyclone fixe est assez réduite, même avec le dispositif de réglage ci-dessus évoqué, on comprend mieux l'intérêt qu'il y a à rechercher des dispositifs permettant de diminuer, sinon de supprimer les fluctuations de toute nature dans ces appareils.

\subsection{Le cyclone tournant}

\section{a) Principe}

La solution développée par Y. Lecoffre et J. Woillez au CERG consiste à faire tourner les parois du cyclone. On élimine ainsi l'essentiel des frottements parasites, et l'on peut obtenir un écoulement réel très proche du vortex théorique. Seuls subsistent des frottements en extrémité du tube qui, pour l'instant, n'ont pu être éliminés, mais dont on peut aisément montrer qu'ils ont peu d'influence sur le fonctionnement de l'appareil dans la gamme granulométrique où il travaille.

Restait à introduire l'émulsion dans le tube tournant. Pour que cette opération se déroule sans choc, il était nécessaire de pénétrer axialement dans l'appareil.

La disposition retenue est schématisée sur la figure 10. L'émulsion provenant de la tuyauterie amont, fixe, passe dans une roue de pompe (3). Cette roue hélico-centrifuge, qui ne doit pas fractionner les gouttes, transporte l'émulsion du repère fixe vers le repère mobile.

Pour obtenir une distribution homogène des vitesses axiales dans l'appareil et supprimer les rotations résiduelles dans le repère tournant, on fait passer l'émulsion dans un espace annulaire cloisonné, disposé à la périphérie du tube tournant (4).

L'essentiel des internes du cyclone tournant est alors quasi identique à ceux du cyclone fixe. On retrouve en effet le cone, permettant de réaliser une divergence progressive de l'écoulement axial, puis le système d'ogive à double tronc de cone et le tube de prélèvement axial dans la zone de sortie.

Quelques ordres de grandeur permettent de préciser les dimensions du prototype :

- Débit nominal : $3 \mathrm{~m}^{3} / \mathrm{h}$

- Diamètre interne : $60 \mathrm{~mm}$

- Longueur : $440 \mathrm{~mm}$

- Vitesse de rotation : de 1500 à $4000 \mathrm{Tr} / \mathrm{mn}$

- Jeu radial cone-cylindre : $5 \mathrm{~mm}$.

Le cyclone mis pour la première fois en essais au CERG en octobre 1985, avait été conçu pour une vitesse nominale de rotation de $1800 \mathrm{tr} / \mathrm{mn}$, soit une vitesse tangentielle en périphérie de $5,65 \mathrm{~m} / \mathrm{s}$, correspondant à une accélération de $100 \mathrm{~g}$.

On notera que l'émulsion pénètre dans le cyclone à la sortie du jeu annulaire, avec une vitesse axiale de $0,8 \mathrm{~m} / \mathrm{s}$, soit un nombre de Reynolds de 4000 . Dans le cylindre, la vitesse moyenne est de $0,3 \mathrm{~m} / \mathrm{s}$. Les fluctuations tur- 
bulentes moyennes peuvent être estimées à $1 \mathrm{~mm} / \mathrm{s}$. Il est prévu de les mesurer par anémométrie laser en 1987.

\section{b) Performances}

Les figures 11 et 12 illustrent le fonctionnement de l'appareil et mettent en évidence la quasi absence de turbulence. L'émulsion, formée de gouttes d'huile difficilement décantables en gravitaire (diamètre moyen $30 \mu$ ) rejoint l'axe du cyclone de telle sorte qu'il se forme une surface de séparation nette entre l'émulsion et le liquide porteur. La même émulsion, traversant un cyclone fixe, est redispersée à mesure que l'on pénètre dans le tube qui reste opaque sur toute sa longueur.

La figure 13 donne l'efficacité de séparation mesurée par comparaison des spectres de gouttes amont et aval. On constate qu'elle est quasi identique à l'efficacité de séparation théorique, basée sur une hypothèse d'écoulement potentiel du fluide porteur. Le modèle de calcul utilisé mériterait encore quelques raffinements. En particulier, il suppose homogène la vitesse axiale dans l'ensemble de l'appareil. L'excellente adéquation de la théorie et des essais montre cependant que la turbulence n’a plus que peu d'influence sur les performances de l'appareil. La figure 14 donne l'évolution de l'efficacité de séparation en fonction du débit. On constate que les résultats expérimentaux restent pratiquement identiques aux prévisions théoriques dans une gamme de débit supérieure à 10 , soit de $0,5 \mathrm{~m}^{3} / \mathrm{h}$ à $5 \mathrm{~m}^{3} / \mathrm{h}$.

On notera également que l'efficacité de l'appareil reste élevée, en-deçà du diamètre de coupure absolu pour lequel $100 \%$ des gouttes sont éliminées. Cette propriété est caractéristique de tous les décanteurs et provient de la distribution spatiale des gouttes à l'entrée des canaux et à la sortie du cone. Les résultats semblent là aussi conformes aux prévisions théoriques, mais doivent être interprétés avec prudence du fait de l'augmentation extraordinaire de l'accélération lorsque l'on se rapproche du centre du cyclone.

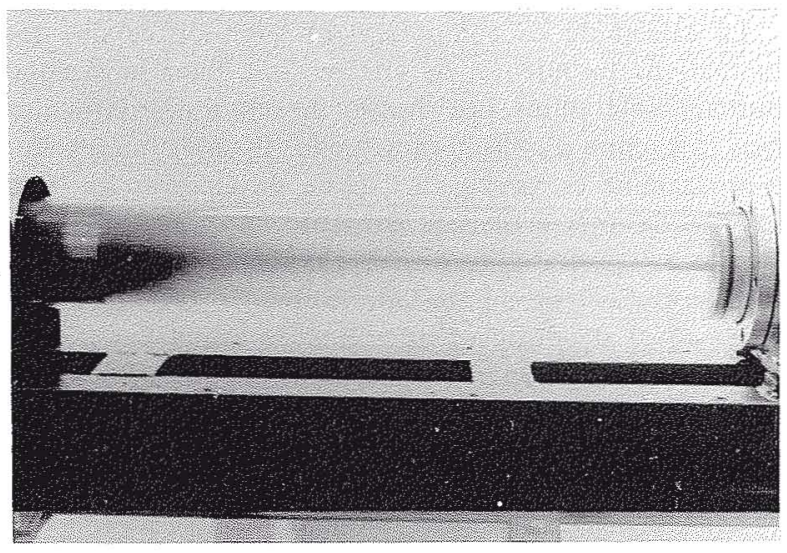

11. Fonctionnement à faible taux dhuile.

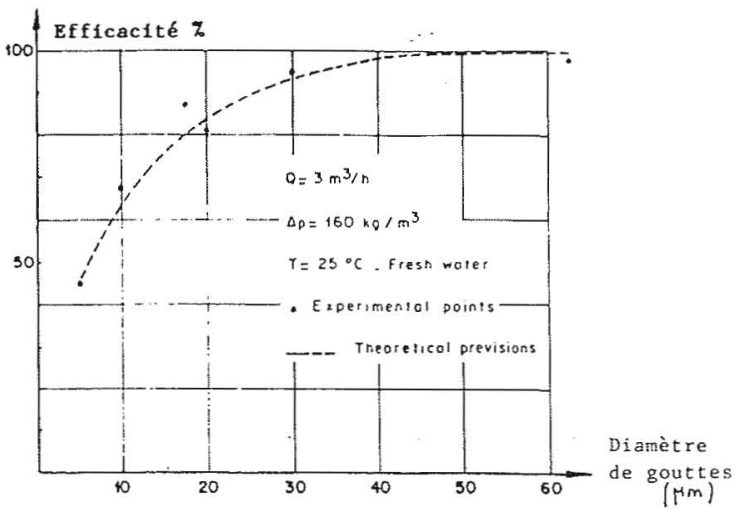

13. Efficacité du cyclone tournant.

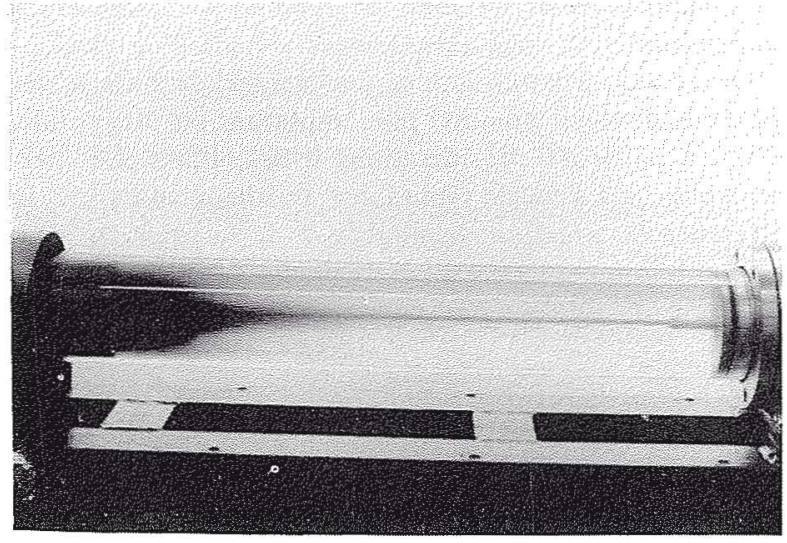

12. Foncrionnement à forl taux dhuile.

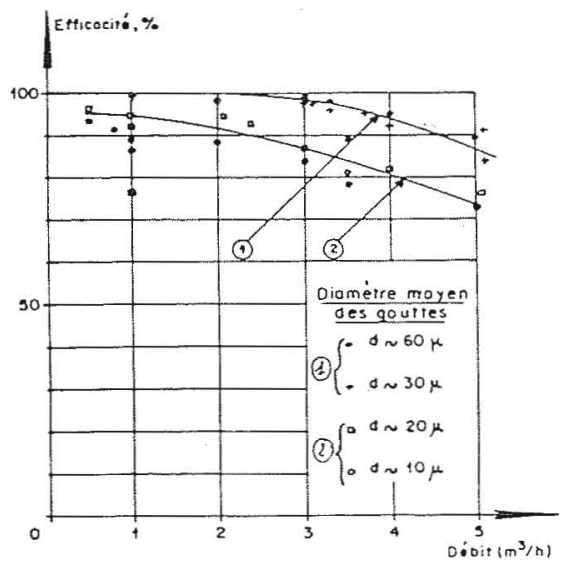

14. Evolution de lefficiacité de séparation en fonction du débit (cyclone tournamit). 
Globalement, le cyclone de $3 \mathrm{~m} / \mathrm{h}$ a des performances pratiquement prévisibles par un modèle d'écoulement potentiel. Les écarts entre théorie et expérience sont inférieurs aux incertitudes de mesure, qui font intervenir, non seulement des concentrations, mais également des distributions granulométriques, dont la connaissance précise demande beaucoup de soins. On a, en particulier, été amené à vérifier systématiquement les indications du granulomètre en les comparant à des macrophotographies des émulsions de microgouttes. On trouvera plus de détails sur la méthode opératoire en référence 3.

\section{c) Extrapolation}

La longueur du cyclone peut être aussi grande que l'on veut. Du fait de l'absence de frottement, la distribution de vitesse est indépendante de la distance à l'entrée. Seul le taux résiduel de turbulence - assorti, bien entendu, de problèmes d'équilibrage éventuels et de balourd - limitera les performances sur une grande longueur.

Il est intéressant de noter que le cyclone tournant se comporte comme une machine hydraulique. On peut aisément, à partir des caractéristiques du prototype de 3 $\mathrm{m} / \mathrm{h}$, par application des invariants de Rateau, prévoir les performances d'un cyclone semblable de dimensions quelconques.

De ce fait, l'extrapolation du cyclone de base à des appareils de débit nominal plus important ou plus faible, se fait à partir de considérations hydrauliques. Les performances maximales réalisables résultent alors de la différence de pression disponible et de la pression aval, qui conditionnent l'apparition de phénomène éventuels de cavitation ou de dégazage dans l'appareil.

\section{d) Développement}

Le premier cyclone a fonctionné en Octobre 1985 et ses performances ont été mesurées sur la boucle d'essai pétrole du CERG à Grenoble (Réf. 4). Divers essais ont été entrepris depuis, dont plusieurs campagnes sur champs et en laboratoires de compagnies pétrolières (ELF, Total).

Un cyclone de débit nominal $25 \mathrm{~m}^{3} / \mathrm{h}$ a été réalisé et testé en laboratoire, puis sur champ pétrolier dans l'émirat de Dubai en novembre 1986 par P. Schummer. Sa longueur était de $2,5 \mathrm{~m}$ et son diamètre interne de $120 \mathrm{~mm}$.

On a pu constater, sur ces différentes installations que les résultats confirmaient ceux du prototype de $3 \mathrm{~m} / \mathrm{h}$, aussi bien en ce qui concerne les performances hydrauliques que les capacités de séparation.

\section{Conclusion}

La turbulence intervient dans tous les organes constitutifs d'un séparateur biphasique en particulier l'entrée et la zone de séparation proprement dite. Autant ses effets sont bénéfiques à l'entrée puisqu'elle tend à homogénéiser la distribution de vitesse, autant elle limite de manière spectaculaire ces performances de la zone de séparation proprement dite.
L'optimisation d'un décanteur gravitaire se fait en tenant compte de la compétition existant entre la vitesse de chute des particules et les fluctuations turbulentes. Lorsque l'on veut réaliser un séparateur à faible diamètre de coupure, on est souvent amené à fonctionner en écoulement laminaire, du fait de la faible accélération disponible.

En écoulement tournant, les champs d'accélération peuvent être aisément multipliés par 10 ou 100 . Bien que, dans les séparateurs traditionnels, les taux de turbulence soient extrèmement élevés, leurs effets sont en partis compensés par les grandes vitesses de chute réalisables. Globalement, les cyclones fixes ont par exemple des performances très supérieures à celles des décanteurs gravitaires, si l'on prend comme facteur de mérite, l'encombrement de l'appareil.

Le cas du cyclone tournant dans ses applications à des émulsions pétrolières traditionnelles, est paradoxal, puisqu'on peut le dimensionner en négligeant totalement la turbulence, jusqu'à des diamètres des gouttes de quelques microns. On obtient en effet expérimentalement les diamètres $d$ de coupure absolus théoriques. On obtient également les courbes efficacité-diamètre prévues par le modèle potentiel pour des globules de dimensions inférieures à $d_{c}$.

La raison de ce comportement vient de ce que les fluctuations turbulentes de vitesse sont, dans le cyclone tournant, inférieures aux vitesses de chute des particules dans la gamme d'utilisation actuelle. Il serait intéressant, le programme est prévu au CERG, de mesurer les fluctuations de vitesse dans le corps de l'appareil et de les corréler aux performances de séparation sur les émulsions très fines. Ceci permettrait de connaître les limites de l'appareil et de disposer de règles de dimensionnement applicables à des appareils de dimensions très différentes. L'extrapolation de 3 à $25 \mathrm{~m}^{3} / \mathrm{h}$ s'est révélée ne pas poser de problème en exploitation. Peut-être n'en serait-il pas de même pour un appareil de $300 \mathrm{~m} / \mathrm{h}$.

\section{Références}

[1] WaLlis. - One dimensionnal two phase flow; Mc Graw-Hill Book, 1966.

[2] ColmaN-THEw. - Correlation of separation from light dispersion hydrocyclone - Chem. Eng. Res. Des., Vol 61, 1983.

[3] Y. Lecoffre, J. Wolllez, J. Genevey. - La boucle d'essai de fluides pétroliers du C.E.R.G. d'Alsthom. Séminaire "Les phénoménes dinterface - Mesure et applications industrielles $»$, novembre 1986.

[4] J. WOILlez, Y. LeCOFfre. - Le cyclone des huileur à parois toumantes - Rapport Neyrtec, novembre 1985.

Adresse de l'auteur

Monsieur $Y$. Lecoffre

Ingénieur conseil

30, nue du Bosc

27590 Pitres Tél. : 32-49-68-38 